\title{
Water assisted injection moulding: development of insights and predictive capabilities through experiments on instrumented process in parallel with computer simulations
}

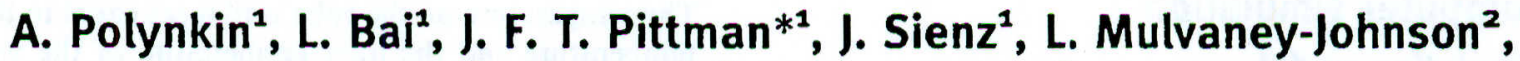 \\ E. Brown ${ }^{2}$, A. Dawson ${ }^{2}$, P. Coates ${ }^{2}$, B. Brookshaw ${ }^{3}$, K. Vinning ${ }^{3}$ and J. Butler ${ }^{3}$
}

An idealised model of core-out in water assisted injection moulding (WAIM) is set up to isolate the effect of cooling by the water on the deposited layer thickness. Based on simulations, this is investigated for a specific case as a function of Pearson number and power law index. It is found that cooling significantly reduces the layer thickness to the extent that a change in the flow regime ahead of the bubble, from bypass to recirculating flow, is possible. For shear thinning melts with high temperature coefficient of viscosity, the simulations show very low layer thickness, which may indicate unfavourable conditions for WAIM. Although in the real moulding situation, other effects will be superimposed on those found here, the results provide new insights into the fundamentals of WAIM. Investigation of other effects characterised by Fourier and Reynolds numbers will be reported subsequently. Some early process measurement results from an experimental WAIM mould are presented. Reductions in residual wall thickness are observed as the water injection set pressure is increased and the duration of water bubble penetration through the melt is determined experimentally. The formation of voids within the residual wall is noted and observed to reduce in severity with increasing water injection pressure. The presence of such voids can be detected by the signature from an infrared temperatures sensor.

Keywords: Water assisted injection moulding, Simulation, Non-Newtonian flow and heat transfer, Instrumented moulding trails

\section{Introduction}

Water assisted injection moulding (WAIM) is an important manufacturing technology with much potential still to be realised. The use of water as the internal fluid offers a number of significant advantages and new possibilities as compared with the now well established gas assisted moulding:

(i) water provides more rapid cooling, reducing cycle times substantially and increasing productivity and energy efficiency

(ii) core-out with water can give parts with thinner walls, further reducing cooling time and also part weight

${ }^{1}$ Centre for Polymer Processing Simulation and Design, $C^{2} E C$, School of Engineering, Swansea University, Swansea, UK

${ }^{2}$ IRC in Polymer Science and Technology, Bradford University, Bradford, (iii) cooling from both inside and outside provides more balanced cooling, resulting in reduced post-ejection warpage

(iv) water core-out can produce larger diameter, longer channels

(v) smooth bore tubular parts with superior internal finish can be produced.

Current and envisaged applications include tubular parts, door handles, roof racks, fascias, bumpers and other automotive components, sports goods, domestic items, etc. The ability to produce complex smooth bore fluid handling ducts, with integrally moulded flanges and other functional elements, provides a very attractive alternative to traditional manufacturing routes. The creation of long, large bore hollow channels offers new possibilities for single step fabrication of large, light weight structures in plastic. These processes are still rather new and the number of applications is relatively small. Full exploitation is hindered by uncertainties 
simulation. Available publications deal with hardware issues and processing conditions. No fundamental studies have been published and no attempt to model the process mathematically has apparently been made. There is very little understanding at a fundamental level of why successful mouldings can be obtained in some cases and in others not.

The present work forms part of a project addressing these issues. In this paper, we report on initial results of computer simulations, which we believe begin to provide insights into some aspects of the physics of the process. An account is also given of parallel experimental work using a highly instrumented test tool for WAIM, with preliminary results.

\section{Computer simulation}

\section{Simulation model}

The displacement of polymer melt by water is modelled from an initially filled tube under simplified, idealised conditions.

\section{Governing equations}

The transient axisymmetric, $v_{\mathrm{r}}(r, z, t), v_{\mathrm{z}}(r, z, t)$, forms of the equations for momentum and incompressible mass conservation are applied for the water and polymer melt, and laminar flow is assumed in both (see further comments below in relation to heat transfer in the water)

$$
\rho(v, \mathrm{t}+v \cdot \nabla v)-2 \nabla \cdot(\mu \dot{\gamma})+\nabla p=0, \quad \dot{\gamma}=\frac{1}{2}\left[\nabla v+(\nabla v)^{\mathrm{T}}\right]
$$

$$
\nabla \cdot v=0
$$

where $\rho, \mu, t$ and $\mathrm{v}$ are density, viscosity, time and velocity vector respectively.

The principle of conservation of thermal energy is written, with $T=T(r, z, t)$, as

$$
\rho C_{\mathrm{p}}\left(\frac{\partial T}{\partial t}+v \cdot \nabla T\right)=\nabla \cdot(k \nabla T)
$$

where $C_{\mathrm{p}}$ and $k$ denote specific heat and thermal conductivity. Viscous heating is omitted. The location of the interface between polymer and water is based on solution of the equation for advection of a fictitious concentration $\psi$

$$
\psi, t+\boldsymbol{v} \cdot \nabla \psi=0
$$

with a high resolution interface capturing algorithm. As the viscosity of the polymer is high, the capillary number is very large and the effects of surface tension can always be ignored; therefore, no special stress conditions are required on the interface, and the water and polymer filled regions are treated as a single domain. The viscosity of the polymer melt is modelled by a temperature dependent power law

$$
\mu=\mu_{0} e^{-\mathrm{b}\left(\mathrm{T}-\mathrm{T}_{0}\right)} \dot{\gamma}^{\mathrm{n}-1}
$$

Thermal diffusivity and density of the plastic are taken to be constant. Physical properties of the water, a Newtonian liquid, are also taken to be constant.

\section{Initial and boundary conditions}

The tube, of length $L$ and radius $a$, is initially filled with polymer melt at temperature $T_{\text {poly }}$

$$
t \leqslant 0, \quad 0 \leqslant r<a, \quad 0 \leqslant z \leqslant L, \quad T=T_{\text {poly }}
$$

At time zero, water begins to penetrate into the tube. An inlet velocity $v_{\text {in }}$ is specified

$$
t>0, \quad 0 \leqslant r<a, \quad z=0, \quad v_{\mathrm{r}}=0, \quad v_{\mathrm{z}}=v_{\text {in }}
$$

The no slip condition is applied at the tube wall and developed flow conditions at the outlet. The symmetry condition is applied on the axis

$$
\begin{array}{llll}
r=a, & 0 \leqslant z \leqslant L, & v=0 \\
0 \leqslant r<a, & z=L, & v_{\mathrm{r}}=0, & v_{\mathrm{z}, \mathrm{z}}=0 \\
r=0, & 0 \leqslant z \leqslant L, & v_{\mathrm{r}}=0, & v_{\mathrm{z}, \mathrm{r}}=0
\end{array}
$$

The temperature on the tube wall is set equal to the melt temperature and the inlet temperature of the water to $T_{\text {water }}$

$$
\begin{aligned}
t>0, \quad 0<r \leqslant a, \quad z & =0, \quad T=T_{\text {water }} \\
z & =L, \quad \partial T / \partial z=0 \\
r & =a 0<z \leqslant L \quad T=T_{\text {poly }}
\end{aligned}
$$

Furthermore, the temperature throughout the water is fixed at $T_{\text {water, }}$ artificially suppressing temperature variations due to heat transfer. This effectively applies the Dirichlet boundary condition at the water/polymer interface $\Gamma_{w / p}$

$$
T=T_{\text {water }} \text { on } \Gamma_{\mathrm{w} / \mathrm{p}}
$$

The reason for these artificial temperature conditions and the neglect of viscous heating is to simplify the problem, allowing examining separately the effect of heat loss from the polymer to the vater. Setting the tube wall temperature equal the polymer temperature suppresses heat loss to the wall. Fixing the water temperature to a uniform value removes complications due to heat transfer within the water, removing the need to model details of the water flow. For computational convenience, the water flow is therefore assumed to be laminar.

\section{Dimensionless quantities}

Interest centres on the thickness of the residual wall layer of plastic $d$ left behind after water penetrates the tube. This is made dimensionless as $\delta=d / a$. The authors are interested in the influence upon this of the melt viscosity increase ahead of the water bubble resulting from heat transfer into the water. This can be characterised by the Pearson number, the product of the temperature coefficient of viscosity with the temperature driving force for heat transfer.

$$
P n=b\left(T_{\text {poly }}-T_{\text {water }}\right)
$$

For shear thinning melts, the power law index $n$ provides a second parameter of interest. It can be anticipated that further relevant dimensionless parameters will include Fourier or Graetz number(s) characterising the heat transfer and Reynolds number(s) related to inertial effects. In the present work, these are not varied and results are presented as a function of $P n$ and $n$ for a typical specific case, to provide initial insights. Dependence on other dimensionless quantities will be explored in further work. 

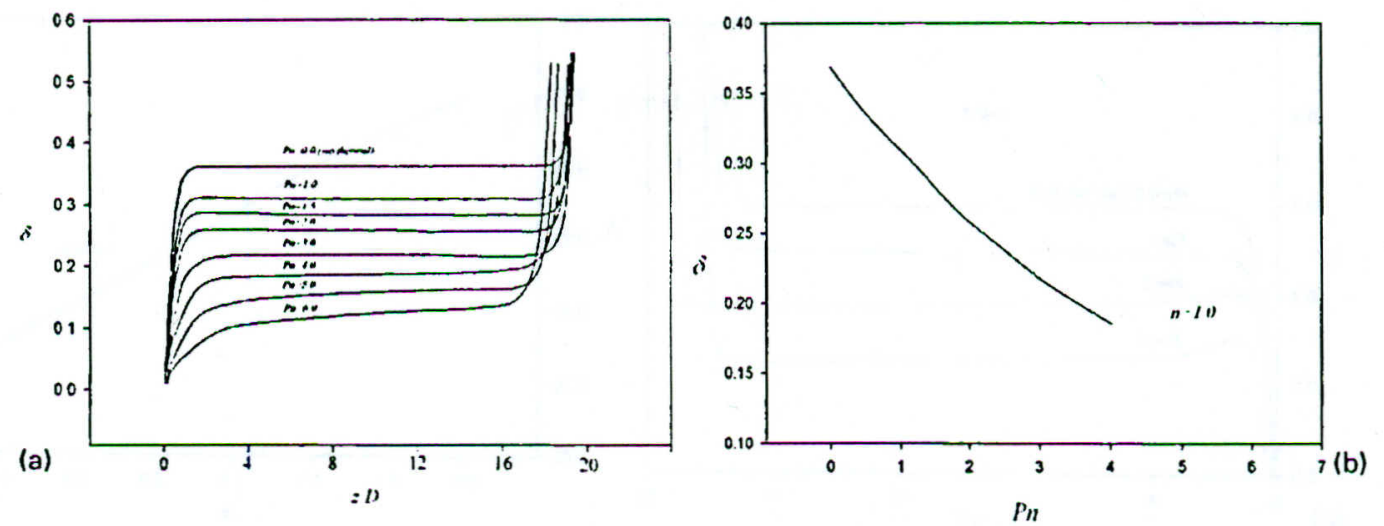

a as function of dimension less axial position with $P n$ as parameter; $b$ steady value of $\delta$ as function of $P n$ 1 Dimensioniess residual layer thickness as function of Pearson: Newtonian melt $n=1$

\section{Computation}

Simulations are carried out using the finite volume package, Fluent, with a structured mesh of quadrilaterals with 40 cells across the tube radius and an axial to radial aspect ratio of $2: 1$, giving a total of 32000 cells. This level of discretisation was chosen after preliminary studies for convergence, smoothness of solutions and agreement with existing literature results for isothermal displacement. The Fluent's volume of fluid method was used with the modified high resolution interface capturing scheme. Euler explicit time stepping was used, with variable time step size controlled by specifying Courant number equal $0 \cdot 25$. Computing time per run was $\sim 4 \mathrm{~h}$ on a single processor AMD Opteron 240 (Linux-64).

\section{Results and discussion Physical property values}

Computations were carried out in dimensional form and results then expressed in dimensionless terms. The following physical property values were used. For polymer melt: $\mu_{0}=150 \mathrm{~Pa} \mathrm{~s}^{\mathrm{n}}, T_{0}=50^{\circ} \mathrm{C}$, thermal conductivity $k=0.3 \mathrm{~W} \mathrm{~m}^{-1} \mathrm{~K}^{-1}$, specific heat $C_{\mathrm{p}}=2.0 \mathrm{~kJ} \mathrm{~kg}^{-1} \mathrm{~K}^{-1}$, density $\rho=1000 \mathrm{~kg} \mathrm{~m}^{-3}$. For water: viscosity $\mu=0.001 \mathrm{~Pa} \mathrm{~s}, k=0.6 \mathrm{~W} \mathrm{~m}^{-1} \mathrm{~K}^{-1}$, $C_{\mathrm{p}}=4 \cdot 182 \mathrm{~kJ} \mathrm{~kg}^{-1} \mathrm{~K}^{-1}, \rho=998 \mathrm{~kg} \mathrm{~m}^{-3}$. Specified temperatures were $T_{\text {poly }}=200^{\circ} \mathrm{C}, T_{\text {water }}=50^{\circ} \mathrm{C}$. The melt temperature coefficient $b$ was varied to provide Pearson numbers in the range $0-6$. Tube radius $a=0.0025 \mathrm{~m}$ and length $L=0.100 \mathrm{~m}$. Water inlet velocity was $v_{\text {in }}=0.1 \mathrm{~m} \mathrm{~s}^{-1}$.

\section{Influence of Pearson number}

In a first set of results, a Newtonian melt is considered, $n=1$. Figure 1 shows the dimensionless residual layer thickness $\delta$ as a function of dimensionless axial position for a range of Pearson numbers. $P \boldsymbol{n}=0$, where no temperature induced change of viscosity occurs, is equivalent to the isothermal case. Results for this case are well known ${ }^{1}$ and are closely reproduced here. After an entry length of about two diameters, $\delta$ attains a steady value until the bubble front is approached. It can be seen that as the Pearson number rises to $\sim 2$, the development length remains similar, but the steady value of $\delta$ falls significantly. For higher Pearson numbers, the development length increases and $\delta$ continues to fall. For $P_{n}=4$ and above, a steady value of $\delta$ is not attained over an axial distance of twenty diameters.

\section{Influence of power law index}

It is well established for the isothermal case that the residual layer thickness decreases with increased degree of shear thinning (lower $n$ ). In Ref. 1, the relationship was correlated by the following empirical form which closely represented the authors' computed results and those in the literature (see Ref. 1 for a review)

$$
\delta=\frac{0.4039 n}{0.08646+n}
$$

In the present situation, the reduction in $\delta$ is enhanced by the effect of cooling around the bubble front. Figure 2 shows a set of plots for $n=0 \cdot 3$. Again, the $P n=0$ result closely reproduces the isothermal case according to equation (12) and $\delta$ falls as $P n$ increases. For $P n=2 \cdot 0$ and above, a steady value of $\delta$ is barely attained over 20 diameters, but continues to fall after an initial overshoot. Results for $P n=4 \cdot 0$ (not shown here) showed irregularities in the wall thickness and it is not yet clear whether this is a physical or computational effect.

\section{Flow patterns ahead of bubble}

The pattern of results shown above appears quite complex, in particular the qualitative change in behaviour at higher Pearson numbers. Insights can be gained by considering the flow patterns ahead of the water bubble, which are most easily visualised in a frame of reference moving with the velocity of the bubble tip. It is well known that the flow pattern depends upon the relative velocities of the bubble and the filament of liquid on the axis in the developed tube flow ahead of it. If velocity on the axis is greater than the bubble velocity, then recirculation occurs ahead of the bubble; if it is less, the streamlines divide around the bubble in bypass flow. The behaviour is governed by continuity requirements and the transition between the types of flow can be expressed in terms of the residual layer thickness, or the fraction $m$ of the tube cross-section occupied by the layer

$$
m=2 \delta-\delta^{2}
$$

In Ref. 1, a criterion was derived applying for power law liquids in terms of a critical value $m^{\prime}$. This is applicable here because developed isothermal flow exists ahead of the bubble 

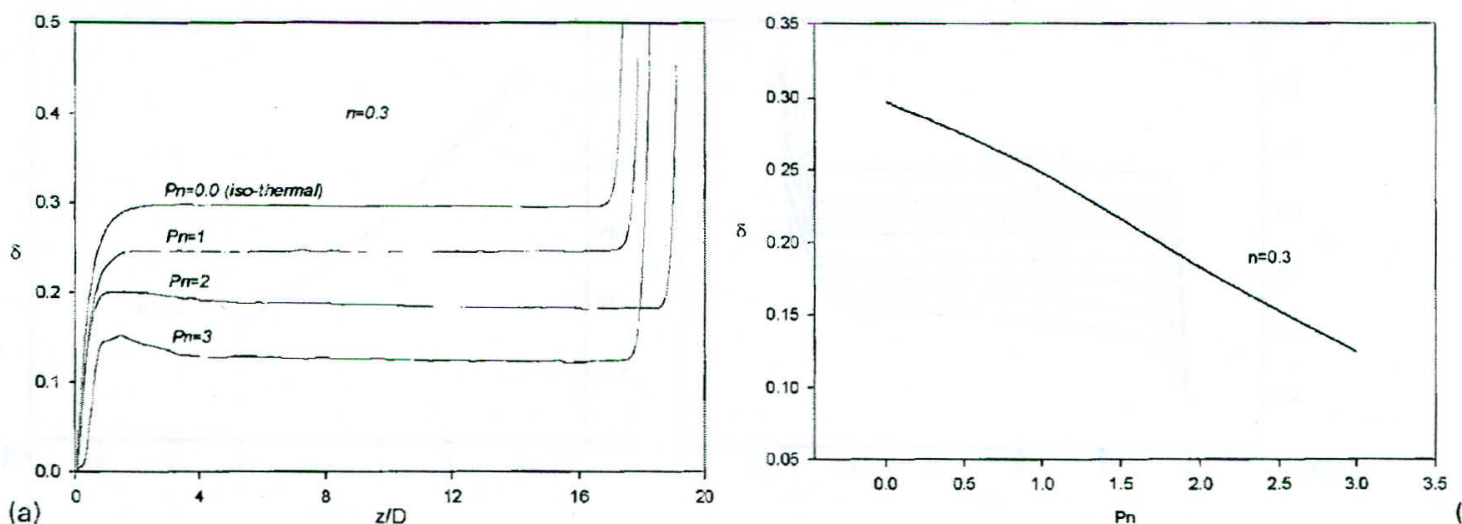

$a$ as function of axial position with $P n$ as parameter, $b$ steady value of $\delta$ as function of $P n$

2 Dimensionless residual layer thickness as function of Pearson: shear thinning melt $n=0.3$

$$
\begin{aligned}
& m^{\prime}=\frac{2 n}{3 n+1} \\
& m<m^{\prime} \quad \text { recirculation } \\
& m>m^{\prime} \text { bypass flow }
\end{aligned}
$$

For the isothermal case, it was found ${ }^{1}$ that the residual layer thickness was always sufficient to produce bypass flow. Here, the reduction brought about by cooling at the bubble front is sufficient to bring about a transition to recirculating flow (Fig. 3). For the Newtonian flow, the transition occurs at $m^{\prime}=0.5$ and this value is reached between $P_{n}=1.0$ and 1.5 . The figure shows how the recirculation continues to develop as the Pearson number rises further and $m$ falls.

Figure 4 shows the corresponding information for shear thinning melt, $n=0 \cdot 3$. Note the flatter shape of the bubble front, which is as previously observed for the isothermal case.

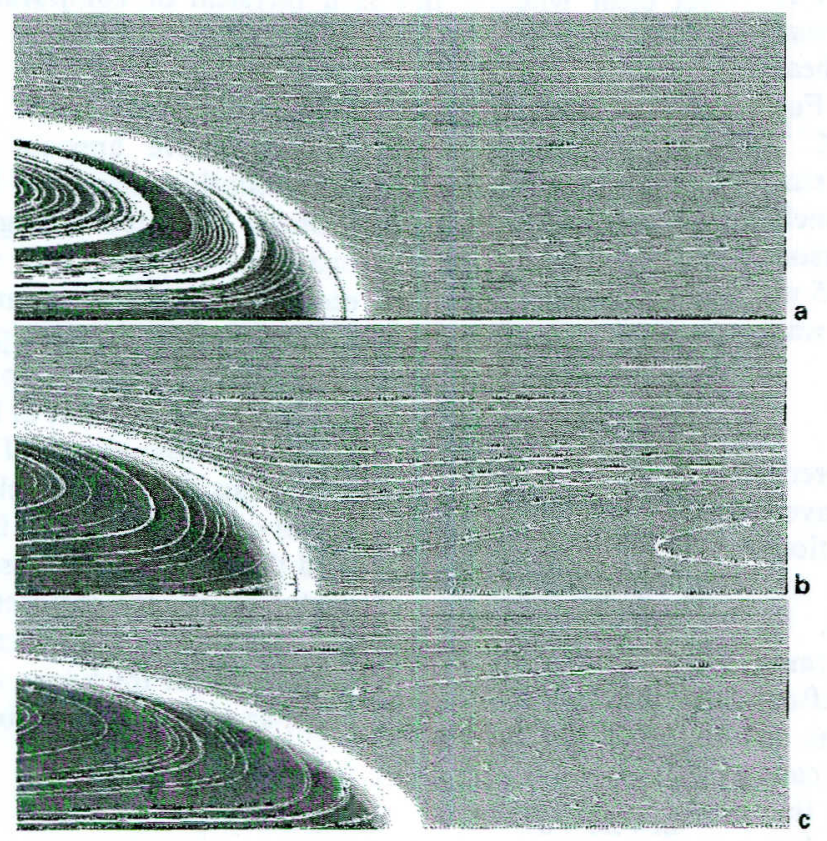

a $P_{n}=1.0, n=1.0, m=0.523 ; b \quad P n=1.5, n=1.0, m=0.486$; c $P_{n}=4 \cdot 0, n=1 \cdot 0, m=0.342$

3 Streamline patterns ahead of bubble in frame of reference moving with bubble velocity: Newtonian melt; contours represent temperature $50-200^{\circ} \mathrm{C}$
The transition in the flow regime explains the qualitatively different behaviour observed at higher Pearson numbers. Figure 5 shows how for the Newtonian case, for $P n=1.5$ and above, recirculation advects material away from the front, extending a region of cooler, more viscous melt a long way ahead of the bubble. Development of this is slow, resulting in the failure to reach steady conditions (relative to the bubble front) within the simulated flow length.

\section{Influence of heat transfer at tube wall}

The results shown above exclude heat transfer to the tube wall, to examine separately the influence of cooling to the internal water. Of course, in the real case, cooling to the wall will occur to a greater or lesser extent and this will tend to increase the deposited layer thickness (see Ref. 2). As a brief indication of this, Fig. 6 shows a comparison between cases where no heat transfer to the wall occurs $\left(T_{\text {wall }}=200^{\circ} \mathrm{C}\right)$ and where cooling to the wall at $T_{\text {wall }}=50^{\circ} \mathrm{C}$ takes place. The influence of this depends on the cooling time, which in turn depends on the injection velocity. In the example shown, a dimensionless cooling time $\tau_{\text {cool }}=\kappa L /\left(a^{2} v_{\text {in }}\right)=4 \cdot 8 \times 10^{-5}$. The layer thickness increases with downstream position as the time available for cooling increases. Note that in the case modeled, the tube is initially filled with melt. This corresponds to the full shot with overflow processing option. If a delay is introduced between melt filling and water injection, the effects of cooling to the wall will, of

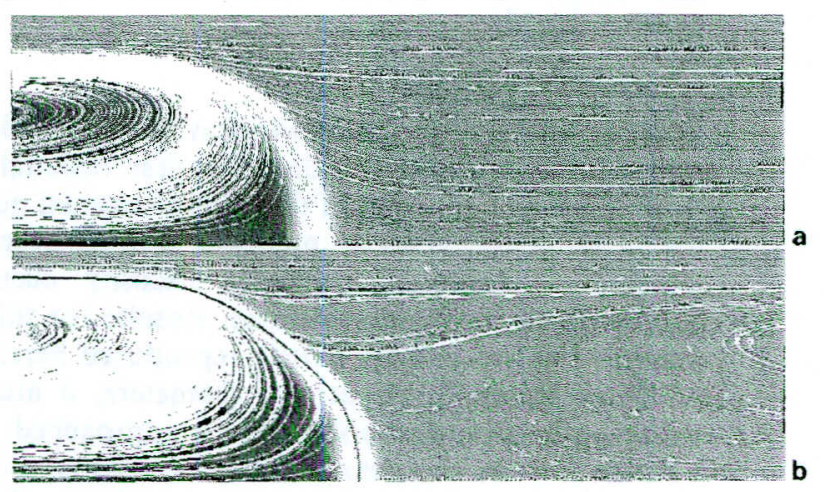

a $P_{n}=1.0, \quad n=0.3, \quad m=0.348 \quad\left(>m^{\prime}=0.316\right) ; \quad b \quad P_{n}=2.0$, $n=0.3, m=0.219\left(<m^{\prime}=0.316\right)$

4 Streamline patterns ahead of bubble in frame of reference moving with bubble velocity: shear thinning melt, $n=0.3$; contours represent temperature $50-200 . \mathrm{C}$ 
$P_{n}$
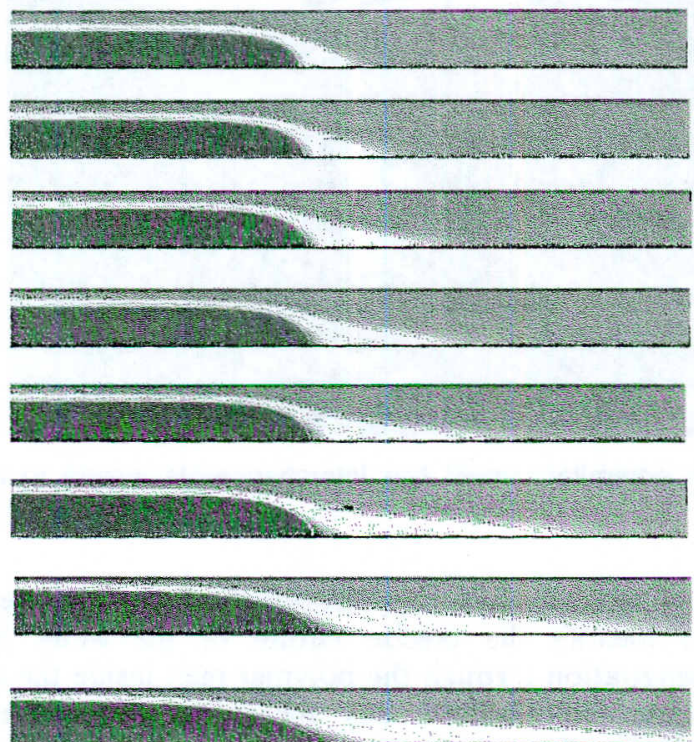

5 Temperature field ahead of bubble: $n=1$; axial position of bubble front $z(2 a)=10$

course, be increased. In short shot processing, the tube is initially partially filled and as water displaces melt down the tube, fresh, hot material is brought to the wall by fountain flow at the melt front. In this case, the effect of cooling to the wall will be less than that shown.

\section{Summary of conclusions from modelling investigations}

A simplified model of core-out in water assisted injection moulding has been simulated to provide insights into fundamental aspects of the process. Cooling heat transfer to the tube wall and viscous heating in the melt have been eliminated, as has been the dependence of the internal heat transfer on details of the water flow. The melt viscosity has been represented by a temperature dependent power law, incorporating a single temperature coefficient, allowing the effect of cooling upon the viscosity around the bubble front to be characterised by a limited number of parameters. The thickness of the (dimensionless) residual laycr thickness $\delta$ has been investigated as a function of Pearson number (which characterises the influence of the internal heat transfer on melt viscosity) and power law index (which characterises melt shear thinning) for a specific, typical case, to provide initial insights. Investigation of the dependence on other dimensionless parameters will be reported subsequently. The idealisations of the model mean that the case $P_{n}=0$ is equivalent to isothermal operation, for which there are well established results that provide a useful baselinc for the present work. The computations closely reproduce these. As the Pearson number rises, the increase of viscosity around the bubble front due to cooling causes a significant decrease in $\delta$. For the Newtonian melt, for $P n$ up to $\sim 3, \delta$ reaches a steady value after a development length of about two diameters. At $P_{n}=1, \delta$ has fallen from the isothermal value of 0.37 to 0.31 . At $P n=2$, it is 0.26 and at $P n=3$, it is $0 \cdot 22$. With a further increase in $P n, \delta$ continues to fall, but the development length is substantially extended so that a steady value is not reached over 20 diameters.

Shear thinning is known to reduce the residual layer thickness; and here, combined with the effect of cooling, very low values of $\delta$ are predicted. For $P_{n}=0.5$ and $n=0 \cdot 3, \delta$ has fallen from the isothermal value of 0.31 to $0 \cdot 28$. For $P_{n}=3$, the low value $0 \cdot 12$ is reached after an initial overshoot.

A significant finding is that the layer thickness falls sufficiently to bring about a transition in the flow pattern ahead of the bubble. In isothermal flow, or at low $P n$, bypass flow occurs. In a frame of reference moving with the bubble front, flow approaches the bubble and divides around it. At higher $P n$, where $\delta$ is lower, recirculation occurs ahead of the bubble. The transition is governed by a critical fractional coverage value $m^{\prime}$, which is a function of $n$. Recirculating flow advects material away from the bubble, producing a long region of cooled, more viscous material ahead of it and slowing the attainment of steady conditions (relative to the bubble front).

To put these predictions into context, consider a typical situation where $T_{\text {poly }}=200^{\circ} \mathrm{C}$ and $T_{\text {water }}=50^{\circ} \mathrm{C}$. Temperature coefficients of viscosity $b$ for thermoplastics can range from $\sim 0.015$ to $0.03{ }^{\circ} \mathrm{C}^{-1}$, with the higher value corresponding to a temperature sensitive material such as polystyrene. The corresponding range of $P_{n}$ is then 2.25-4.5 and it seems possible that the recirculating flow regime may therefore exist in practical molding situations. It can be speculated that this may be

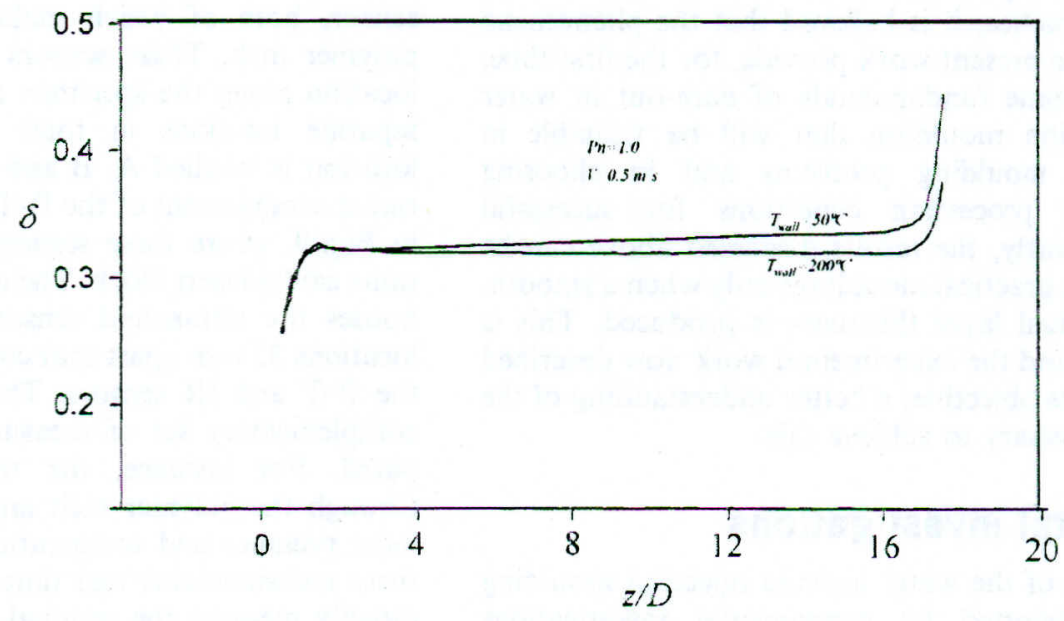

6 Effect on residual layer thickness of cooling to tube wall 


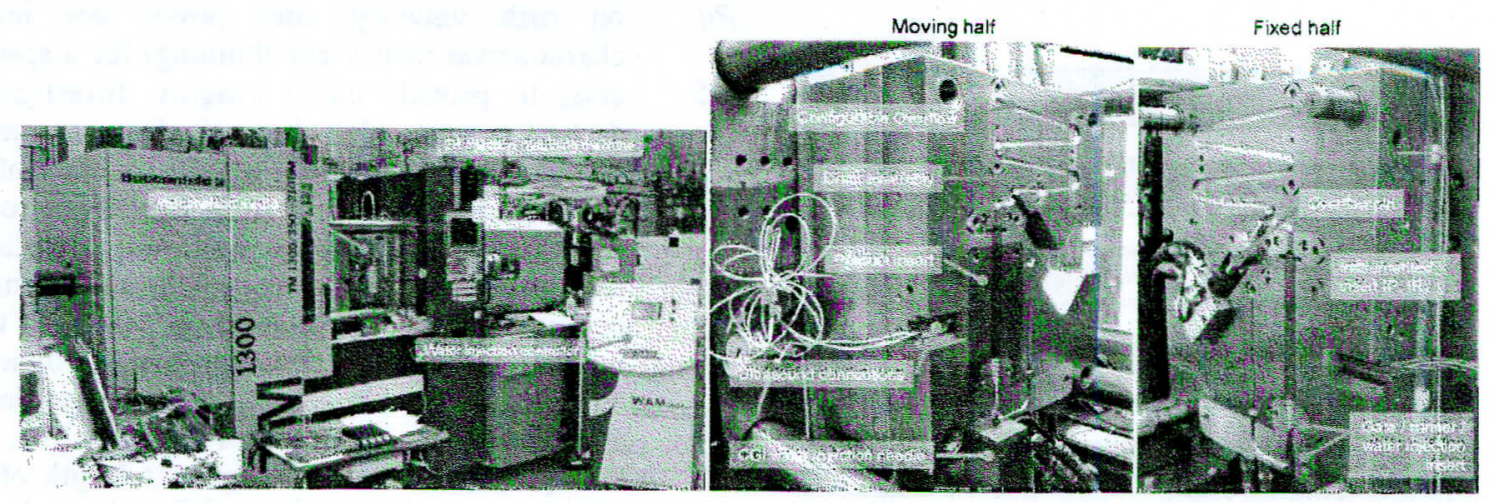

$72 \mathrm{~K}$ injection moulding machine fitted with CGI water injection controller: mould has interchangeable inserts to investigate difference geometry and process configurations

a less stable regime for core-out. Furthermore, for a highly temperature sensitive, strongly shear thinning material, very low residual layer thickness is predicted, possibly with the presence of irregularities. Again, this may not be a satisfactory moulding situation. It has been suggested in the literature that increasing water temperature can sometimes be helpful in water assisted molding, and the present results may indicate why this is so (it reduces $P n$ ).

The model analysed here is idealised and the quantitative predictions are not expected to be accurate for real mouldings. In particular, heat transfer to the cavity wall will increase viscosity there and give a larger residual layer thickness. This suggests that for a highly temperature sensitive, shear thinning material, the possible difficulties mentioned above may be overcome by delaying water injection to allow some heat transfer to the wall. The idealisation of a uniform water temperature represents an upper limiting case for heat transfer. In reality, the cooling effect will be less intense and the residual layer thickness will be somewhat larger. However, fountain flow in the water at the bubble front will provide strong cooling. Extrapolation of viscosity down to low temperatures using the exponential form with a single temperature cocfficient will often be unrealistic and definitely so for semicrystalline materials. The indications are that a more rapid viscosity increase below the no flow or solidification temperature will further amplify the layer thickness reduction due to internal cooling.

Although the quantitative predictions may not be born out in practice, it is believed that the phenomena identified in the present work provide, for the first time, insights into some fundamentals of core-out in water assisted injection moulding that will be valuable in understanding moulding problems and in choosing materials and processing conditions for successful operations. Clearly, the results discussed above can be compared with practical mouldings only when a smooth, void free residual layer thickness is produced. This is often difficult and the experimental work now described has as one of its objectives a better understanding of the conditions necessary to achieve this.

\section{Experimental investigations}

The modelling of the water assisted injection moulding process is supported by experimental investigations through an instrumented injection moulding machine and tooling system. Key process measurements are made to identify the precise nature of the water bubble penetration through the polymer melt inside the mould cavity. In addition, key measurements of the melt injection from the moulding machine are made such as screw position (therefore melt injection rate and quantity of melt), hydraulic pressure, nozzle melt pressure and nozzle IR melt temperature.

\section{Instrumented tooling system}

The water assisted injection equipment comprises of an injection moulding machine, a water injection controller and a tooling system to form the test specimen (see Fig. 7). The moulding machine is a twin barreled $130 t$ Battenfeld unit with an external connection to the Cinpres gas injection (CGI) water assisted injection control system. The water injection controller can deliver water pressure up to $300 \mathrm{bar}$ and the water pressure output from the system can be controlled to any intermediate pressure for any duration of time. The tooling system has the facility for interchangeable inserts to allow for flexibility in the specimen geometry. The results shown within this work are for a specimen geometry that is tubular with a diameter of $20 \mathrm{~mm}$ and a flow length of $\sim 200 \mathrm{~mm}$. The overflow geometry is a $10 \mathrm{~mm}$ diameter that is variable in flow length through alteration of the insert arrangement.

The in-cavity instrumentation is a key feature of the tooling system that provides important process measurements. Figure 8 shows the cavity wall mounted infrared sensor and combined pressure and temperature (P-T) sensor, both of which make direct contact with the polymer melt. These sensors are mounted in the same location along the specimen and are replicated at three separate locations in total each $35 \mathrm{~mm}$ apart; each location is labelled A, B and C as noted in Fig. 8. The radial arrangement of the P-T and IR sensors are shown in Fig. 9, where these sensors are $90^{\circ}$ apart within the same cavity insert block. The opposing cavity insert block houses the ultrasound sensors that are again in three locations $35 \mathrm{~mm}$ apart that coincide with the positions of the $\mathrm{P}-\mathrm{T}$ and $\mathrm{IR}$ sensors. This arrangement provides a complementary set of measurements that can be compared. For instance, the transit time of ultrasound through the polymer melt and solid is dependant upon local pressure and temperature, therefore having all of these measurements real time provides the capability to directly measure the residual wall thickness of the melt deposited by the advancing water or gas bubble. 

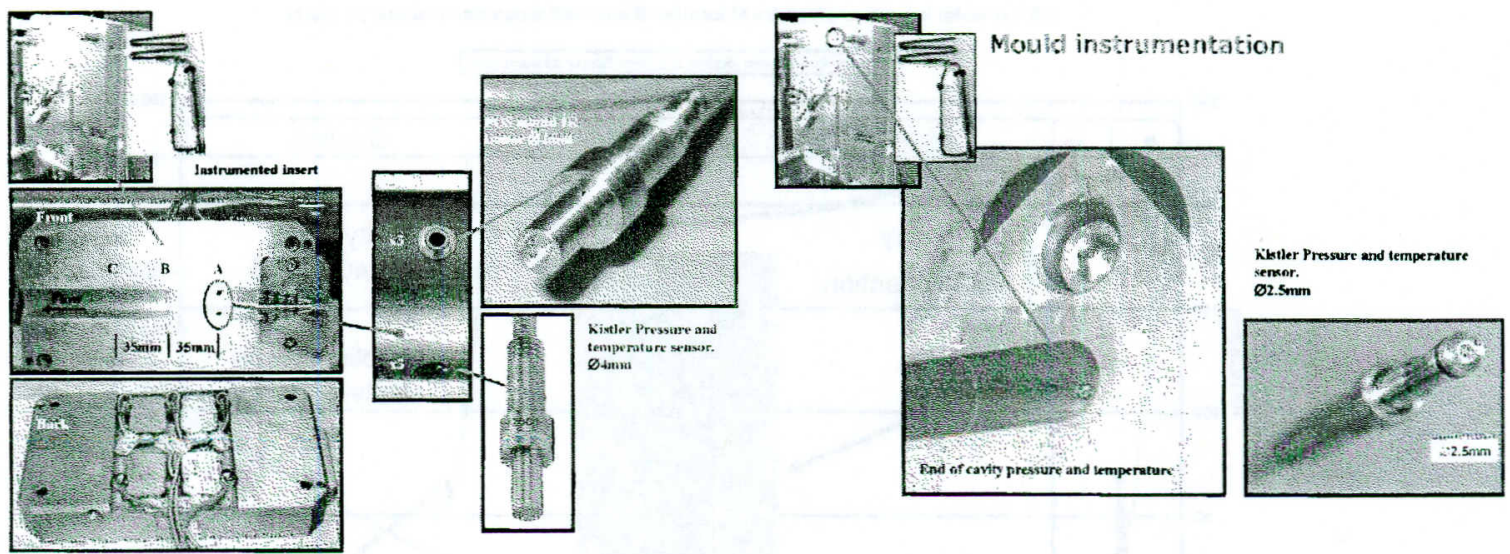

8 Specimen insert has three sites for detailed measurements and each site contains infrared temperature sensor, combined pressure and temperature sensor and ultrasound sensor behind mating cavity insert. In addition, combined pressure and temperature sensor is mounted at end of cavity to detect completion of filling

In addition, a combined pressure and temperature sensor (Fig. 8) is located at the end of the mould cavity, which is used mainly to detect melt arrival and therefore the completion of cavity filling.

\section{Experimental results}

The experimental data presented within this paper is from the processing of a $20 \%$ glass fibre filled polypropylene supplied by A. Shulman polymers (Polyfort FPP 20GF). The water assisted injection moulding process is presented in the form of key process measurements within Fig. 10. Figure $10 a$ shows the various phases of the process against melt injection screw displacement and the cavity pressure measured by the P-T sensor at location B. The first peak on the pressure data $(\sim 1.5 \mathrm{~s}$ of process time) coincides with the end of melt injection during the prefilling of the short shot process. It can be observed that the screw position reaches a minimum at this time. Water injection takes place at $\sim 2.5 \mathrm{~s}$ of process time and an associated rapid rise in cavity pressure can be observed. The water pressure injection set point is 200 bar for the data shown. Figure $10 b$ shows the same pressure data presented in Fig. 10a, but adds the temperature from the thermocouple mounted at the end of the cavity. The sharp rise in this temperature indicated the arrival of

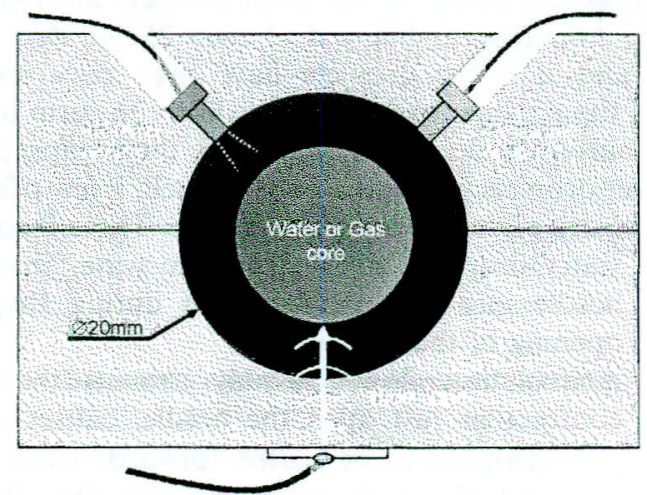

9 There are three sites (A, B and C) where instrumentation is located. This flgure details how pressure, IR and ultrasound sensors are located to track bubble progression and measure local melt pressure and temperature the melt and therefore cavity filling. More significantly, the water bubble penetration will cease at this instant and a sharp rise in the cavity wall pressure is notcd, presumably owing to the reduction in water flow through the injection needle and equalisation of pressure across the needle. Photographs of a short shot specimen without water injection and a specimen containing a fully formed water bubble that has completed cavity filling are shown in Fig. $10 b$.

Since the start and end of water bubble penetration can be determined from the cavity pressure and end of cavity temperature data, the duration of water bubble penetration can therefore be determined. This measurement from these data is highlighted in Fig. $11 a$ for a water injection pressure of $200 \mathrm{bar}$. The water bubble penetration time is noted for water injection pressures of 50, 100, 150 and 200 bar and presented in Fig. 11b. The penetration time at 50 bar of water pressure is $\sim 6.5 \mathrm{~s}$; however, a significant reduction to $1.4 \mathrm{~s}$ is observed at 100 bar and continues to reduce at the water injection pressure increases up to $200 \mathrm{bar}$. The water bubble primary penetration on some samples that have been sectioned is $\sim 650 \mathrm{~mm}$ - this would correlate to an average bubble penetration speed through the polymer melt of 100 and $464 \mathrm{~mm} \mathrm{~s}^{-1}$ at water pressures of 50 and 200 bar respectively.

A bubble velocity measurement could be made directly by use of the ultrasound sensors mounted in three locations. An echo from the bubble front has been observed in gas assisted injection moulding ${ }^{3}$ at two separate US sites and the velocity of the bubble front was therefore determined. At the time of writing, the updated ultrasound system developed and installed on this water assisted mould has not been commissioned, so therefore no data exists to make this direct bubble front velocity measurement.

Specimens have been prepared at the water injection pressures previously noted, $50,100,150$ and 200 bar. Cross-sections of the specimens at the locations A, B and $\mathrm{C}$ at these pressures are presented in Fig. 12. Several samples were manufactured at each pressure and the formation of voids within the residual wall has been noted. The most voids were observed at the lower water injection pressure and the incidence of voids decreased as the water pressure increased up to $200 \mathrm{bar}$, where no voids were observed. 
200 bar water injection: pressure at location $B$ and wall temperature at end of cavity

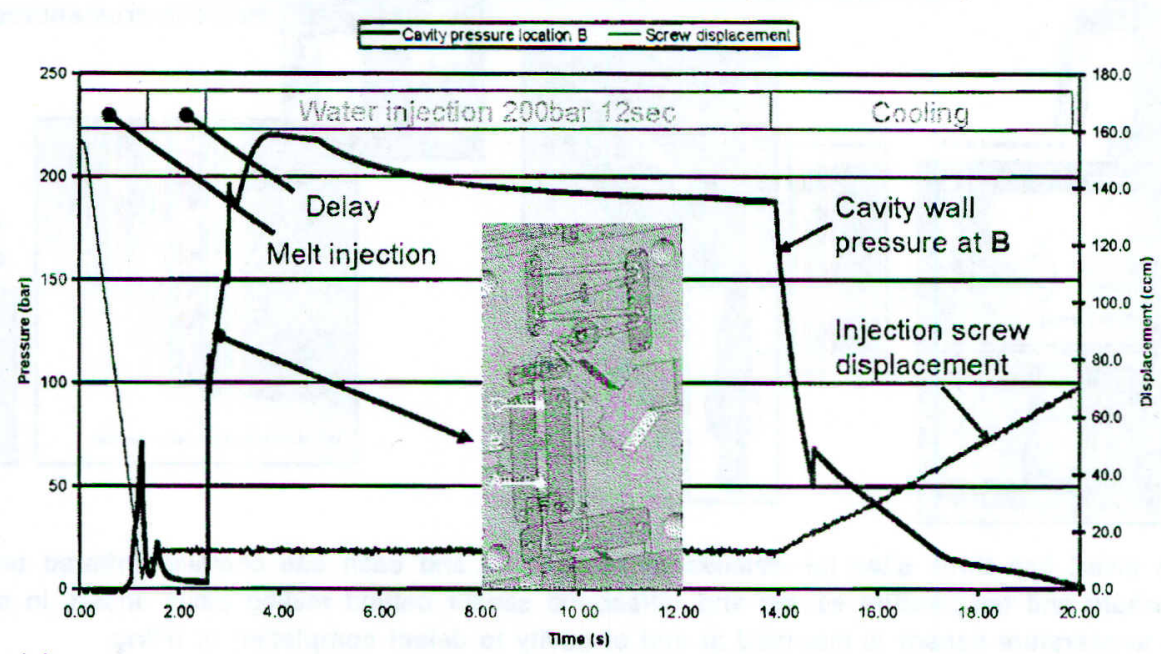

(a)

150 bar waver injection: pressure at location $B$ and wall temperature at end of cavity

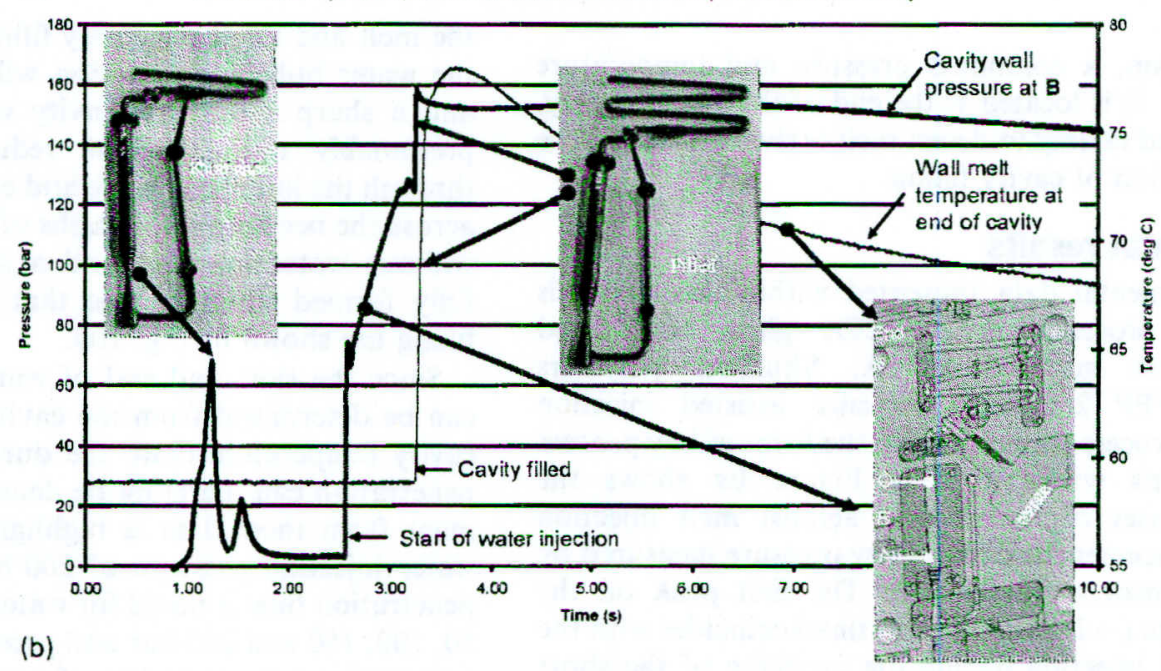

10 Cavity pressure, injection screw position and cavity wall temperature within process cycle: a phases of water injection moulding process are shown; melt pressure is from location $\mathrm{B}$ and melt injection screw position is shown; $b$ short shot of melt is injected into mould before water injection; first peak in cavity pressure is identified and coincides with end of melt injection; next significant rise in cavity pressure is from water injection; wall mounted thermocouple at end of cavity records sharp rise as melt arrives and therefore cavity is filled

The voids forming within the residual wall are thought to be liquid water droplets that turn to steam owing to the high temperature conditions within the mould. The precise nature of this phase change is not known at this time; however, an expansion of the water from the liquid to the gaseous phase is anticipated. Table 1 contains some values extracted from the steam tables that are relevant to the processing conditions experienced by the polymer melt. For instance, a transition from liquid to steam at a constant temperature of $250^{\circ} \mathrm{C}$ and pressure of 40 bar would see a 40 fold expansion in the void volume. A further reduction in pressure at this temperature would see a much increased expansion of the void volume; for instance at $100^{\circ} \mathrm{C}$ and ambient pressure, the void could expand by a factor of 1333. It is clear that a relatively large void that forms within the residual wall could originate from a very small water droplet size that was encapsulated within the polymer melt.

The data presented in Fig. 13 show the cavity pressure at location A and the end of cavity wall melt temperature for a range of water injection pressures. It is clear that for the case where the set water pressure is injected at $50 \mathrm{bar}$, the pressure within the melt at location $B$ is below 40 bar for some of the initial water injection phase. If the local pressure around the water bubble front is below 40 bar at $250^{\circ} \mathrm{C}$, then steam will form and if water droplets have been encapsulated within the polymer melt, a void is likely to form.

If voids are indeed formed through the encapsulation of water droplets within the polymer melt, a few possible theories are currently being considered. These include:

(i) water droplets 'spray' from the water injection needle into the polymer melt during the first opening of the needle

(ii) recirculation flow, possibly around the flow contraction just after the needle, causes water droplets to be pulled forwards from the water/ polymer melt interface and deposited further upstream.

The presence of voids in the residual wall can be detected by the IR temperature sensor that 'looks' some depth into the polymer melt. Figure 14 presents the temperature data from both the IR and themocouple 
(a)
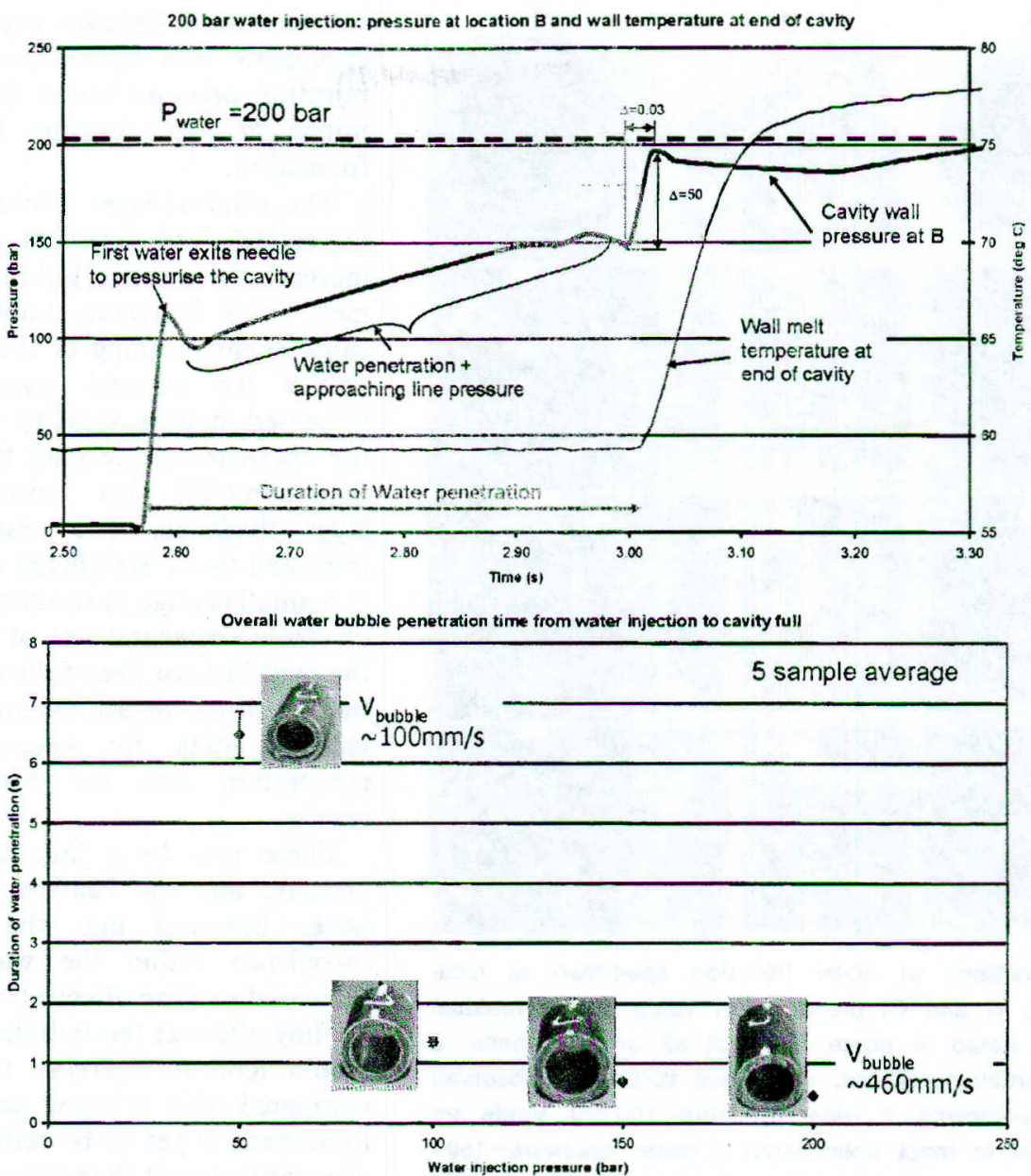

(b)

11 Duration of water bubble penetration: a duration of water penetration during primary filling can be determined; $b$ duration of water penetration at set pressures of $50,100,150$ and 200 bar

sensors for several consecutive water injection cycles, $10-15$, where the water pressure is 50 bar.

Significant void formation is observed, some of which are immediately in front of one of the temperature sensors. The IR data (uncalibrated volts) for cycles 11 and 13 clearly depart from the data of the remaining cycles 10,12 and 14. It appears that the temperature reading from the $I R$ sensor falls more rapidly if a void is present in front of the sensor compared with the presence of a solid residual wall. It may be possible that if the voids are formed by the production of steam, then the rapid fall in temperature could be associated with latent heat of evaporation. It is interesting to note that the thermocouple temperature is largely unaffected by the presence of any void, as in cycle 12 , which supports the assumption that the IR sensor is indeed collecting IR radiation from some depth into the polymer melt and/or solidified material.

Table 1 Saturated steam values (www.efunda.com)

\begin{tabular}{llll}
\hline & & \multicolumn{2}{c}{ Density, $\mathrm{kg} \mathrm{m}^{-3}$} \\
\cline { 3 - 4 } $\begin{array}{l}\text { Temperature, } \\
\text { C }\end{array}$ & $\begin{array}{l}\text { Pressure, } \\
\text { bar }\end{array}$ & $\begin{array}{l}\text { Saturated } \\
\text { liquid }\end{array}$ & $\begin{array}{l}\text { Saturated } \\
\text { vapour }\end{array}$ \\
\hline 250 & $39 \cdot 7$ & 799 & 19.9 \\
200 & 15.5 & 864 & 7.9 \\
100 & 1 & 958 & 0.6 \\
\hline
\end{tabular}

Figure 15 presents the measurement of residual wall thickness for a range of water injection pressure settings at each of the instrumented sites A, B and C. The residual wall thickness tends to decrease as the water pressure increases and this would correlate with an expected increasing water bubble front velocity. An indication of increasing bubble front velocity has already been presented from the duration of cavity filling measurement.

\section{Summary and conclusions from experimental investigations}

The results presented here provide a necessary insight into the mould conditions during the water injection phase of the processing. The instant when the water bubble is first injected into the mould is observed as a rapid increase in cavity pressure and the time taken for the water bubble to progress through the melt to compete cavity filling is noted by a sharp increase in cavity wall temperature at the end of the cavity. The known time for cavity filling provides an opportunity to estimate the average bubble velocity through the melt. At 200 bar of water injection pressure, the average bubble velocity is estimated to be $\sim 464 \mathrm{~mm} \mathrm{~s}^{-1}$, which is greatly higher than the velocity of $100 \mathrm{~mm} \mathrm{~s}^{-1}$ calculated for a water injection pressure of 50 bar.

The formation of voids within the residual wall provides an added complication for drawing conclusions 
A

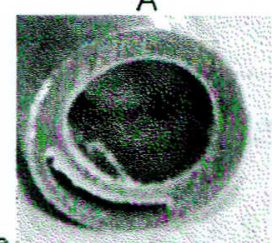

a

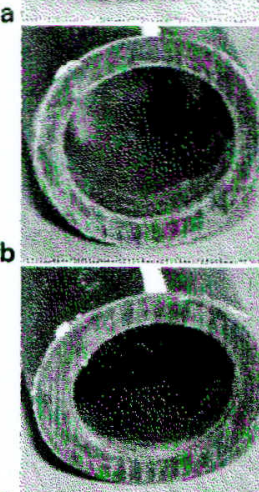

c

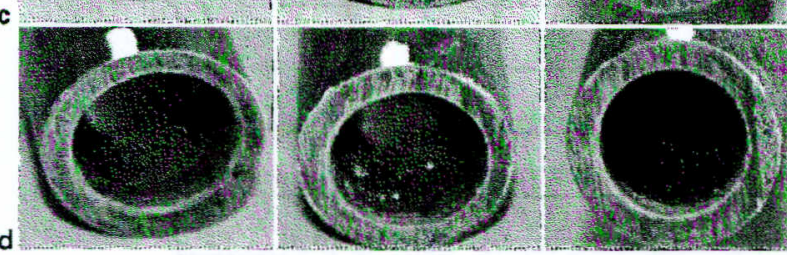

12 Cross-sections of water injection specimen at locations $A, B$ and $C$ : presence of voids within residual wall is noted in some, but not all of specimens: a water pressure $\mathbf{5 0} \mathrm{bar}$, significant voids are observed in all specimens; $b$ water pressure $=100$ bar, voids are observed in most specimens; $c$ water pressure $=150$ bar, voids are observed in a few specimens; $d$ water pressure $=\mathbf{2 0 0}$ bar, none of specimens contain voids in residual wall at any of locations $A, B$ or $C$

from the experimental work presented here. The mechanism for void formation is not fully understood at this time; however it is believed to result from water droplets becoming encapsulated within the polymer melt that subsequently become steam therefore generating a void. Further investigations are necessary to determine whether water droplets are present within the walls of specimens that have been formed using high water injection pressure where the droplets have been maintained at high pressure to prevent significant void formation.

The residual layer thickness appears to reduce with increasing water injection pressure, which is known to increase the average bubble front velocity through the melt. This increased bubble velocity is thought to cause shear thinning of the melt, which would tend to reduce the residual layer thickness. However, the increased bubble velocity would also tend to reduce the characteristic contact time $\tau_{\text {bubble }}$ for heat transfer (equation (12)) that tends to increase the residual layer thickness. The results from the simulations indicated that a significant change in this value resulted in a small change in residual layer thickness. As yet, the ultrasonic measurement of bubble tip velocity through the melt has not been fully commissioned, but it is clear that a direct measurement of this type will provide valuable data for determining the actual bubble penetration rate for comparisons with simulation results.

There may be a link between the water injection pressure and the Pearson number $P n$ in that higher water flowrates into the melt may cause greater turbulence within the water bubble and therefore, increased cooling effect at the bubble tip. An increased cooling effect at the bubble tip and therefore higher $P n$ would tend to decrease the residual layer thickness compared with a lower water injection pressure. This hypothesis is yet to be tested experimentally; however, ultrasonic transit time through the melt that is linked to temperature could provide some useful insights as could the infrared temperature data.

\section{Acknowledgements}

This work forms part of a project supported by the EPSRC, UK with grant EP/D0390021/1. Significant industrial support has been provided by Cinpres Gas Injection Ltd in the form of water injection equipment and design expertise. Materials have been supplied to the

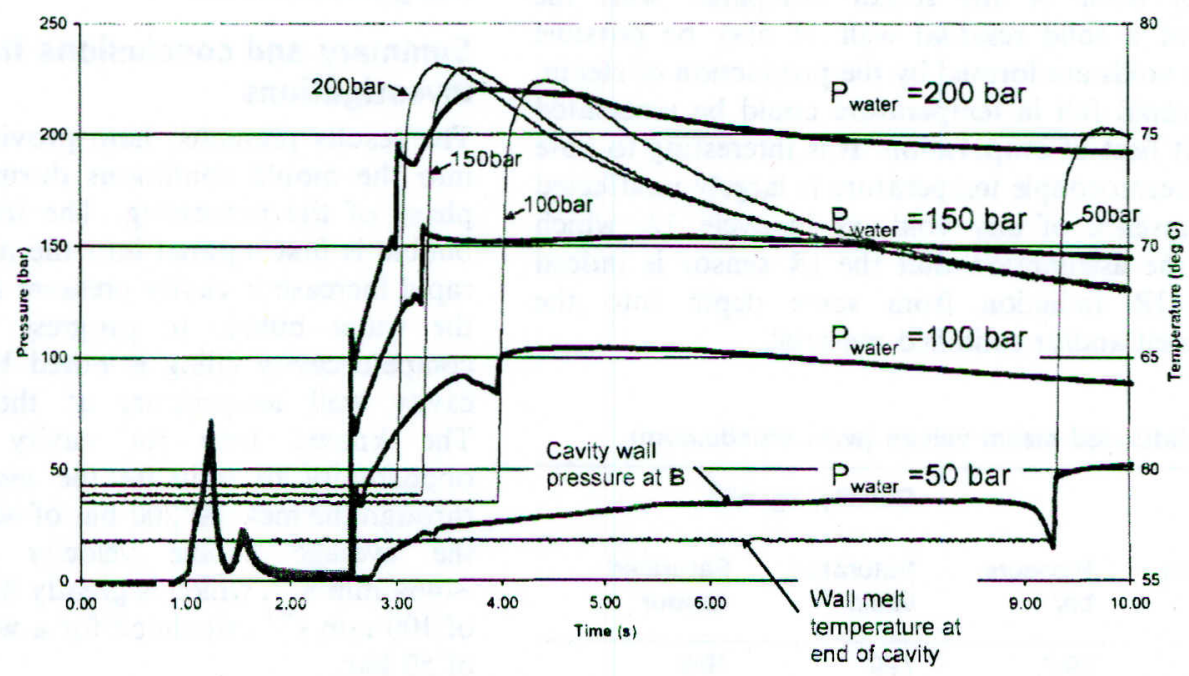

13 Cavity pressure and temperature at end of cavity for range of water injection pressure settings: pressure at location $B$ and wall temperature at end of cavity 

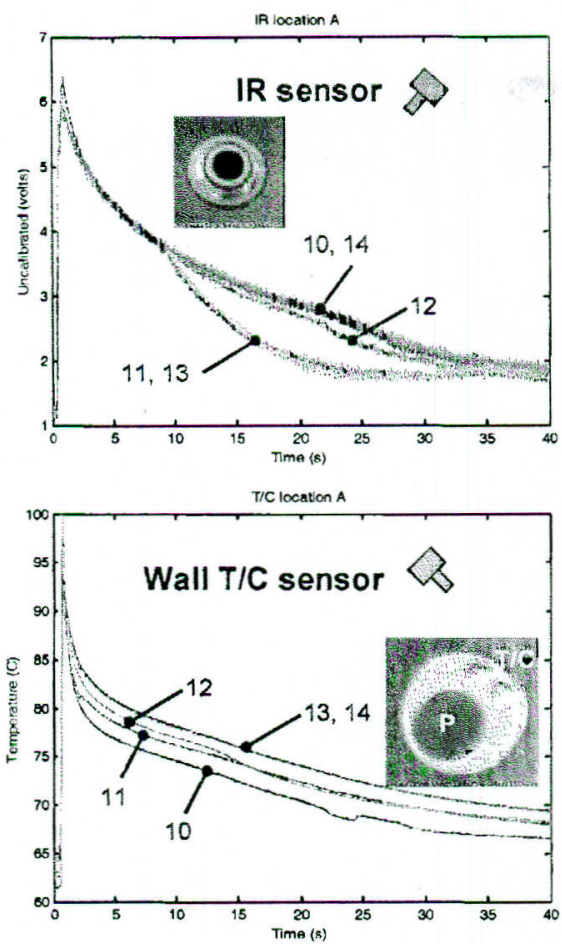
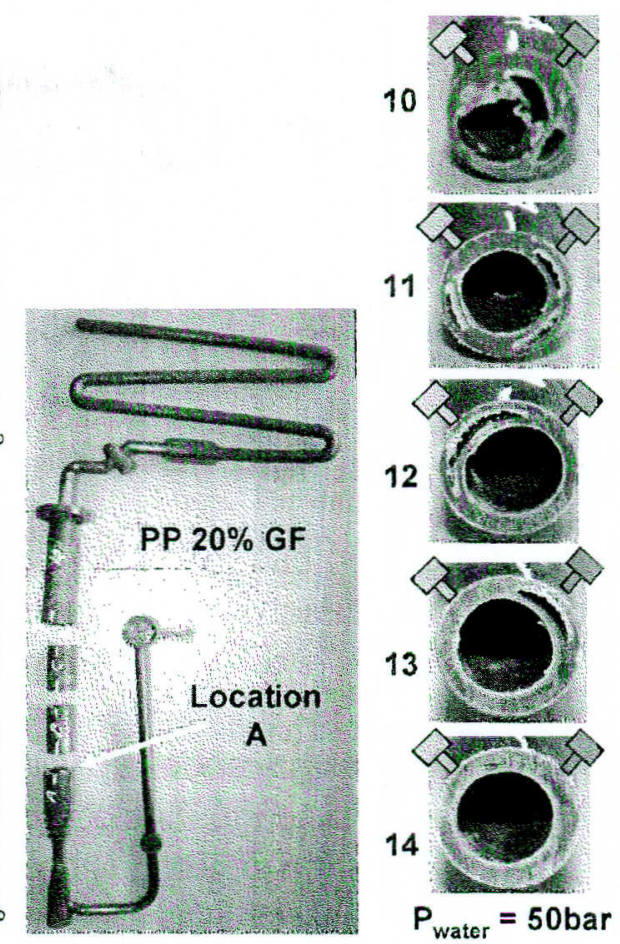

14 Voids within residual wall immediately in front of IR temperature sensor appear to be detected when compared with residual wall that is solid. Voids in front of thermocouple sensor are not clearly detected

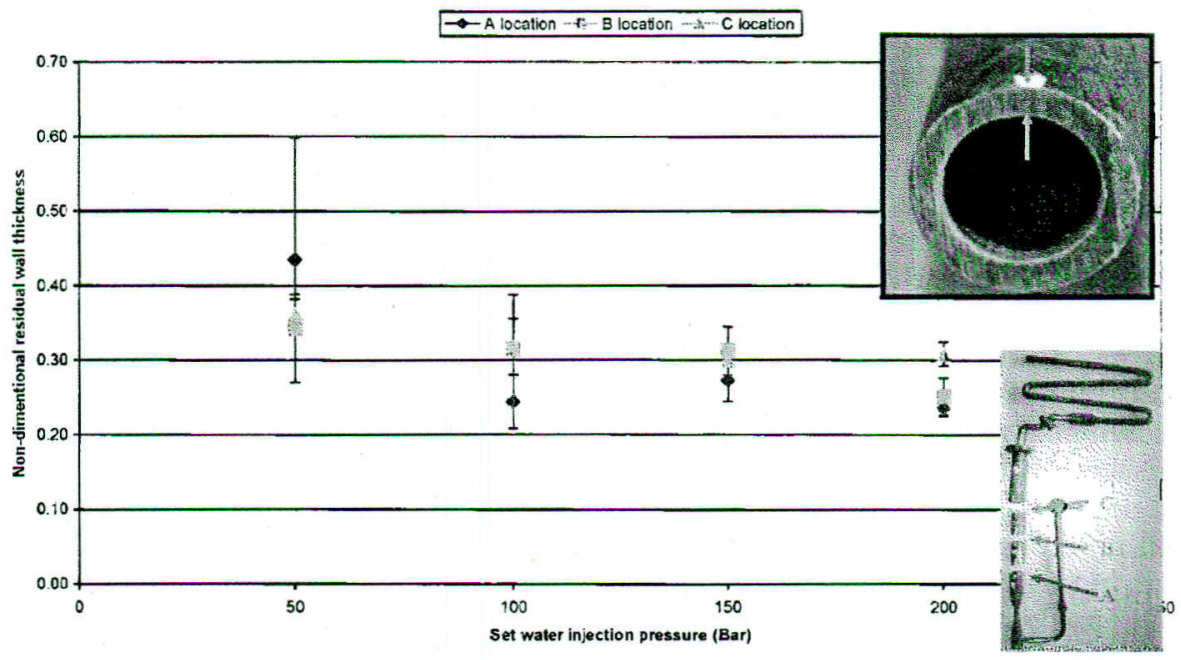

15

Residual wall thickness generally reduces with increased water injection pressure. Non-dimensional wall thickness is expressed as fraction of section radius $(10 \mathrm{~mm})$

programme by A. Schulman Inc. and BASF. Kistler Instruments have both supplied and donated in cavity pressure and temperature sensors; the authors thank Diarmuid Cooper for his time and expertise. This paper is based on a presentation at the Polymer Process Engineering conference held in Bradford, UK in July 2007.

\section{References}

1. A. Polynkin, J. F. T. Pittman and J. Sienz: Chem. Eng. Sci., 2004, 59, 2969-2982.

2. A. Polynkin, J. F. T. Pittman and J. Sienz: Chem. Eng. Sci., 2005, 60, 4953-4956.

3. L. Mulvaney-Johnson, C. C. Cheng, Y. Ono, E. C. Brown, C. K. Jen and P. D. Coates: Plast. Rubher Compos., 2007, 36, 111-121. 